\title{
Propriedades físico-químicas da polpa de tamarillo (Solanum betaceum) e sua aplicabilidade na elaboração de sorvetes
}

\section{Physicochemical properties of tamarillo pulp (Solanum betaceum) and its applicability in the production of ice cream}

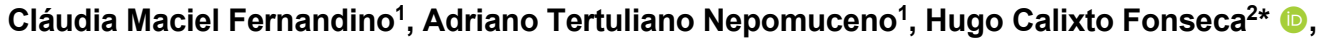 \\ Rejiane Avelar Bastos ${ }^{3}$ (D), Juliana Pinto de Lima ${ }^{4}$
}

${ }^{1}$ Instituto Federal do Sudeste de Minas Gerais, Tecnologia de Alimentos, Barbacena/MG - Brasil

${ }^{2}$ Universidade Federal de Lavras (UFLA), Departamento de Ciência dos Alimentos, Lavras/MG - Brasil

${ }^{3}$ Instituto Federal do Sudeste de Minas Gerais, Ciência de Alimentos, Barbacena/MG - Brasil

${ }^{4}$ Universidade Federal de Minas Gerais (UFMG), Instituto de Ciências Agrárias, Montes Claros/MG - Brasil

${ }^{*}$ Corresponding Author: Hugo Calixto Fonseca, Universidade Federal de Lavras (UFLA), Departamento de Ciência dos Alimentos, Caixa Postal: 3037, CEP: 37200-900, Lavras/MG - Brasil, e-mail:

hugocfonseca@yahoo.com.br

\begin{abstract}
Resumo
O tamarillo, também conhecido no Brasil como tomate de árvore, tem ganhado projeção devido à riqueza de nutrientes e compostos bioativos que apresenta, sendo um candidato promissor para aplicação pela indústria alimentícia. Ressalta-se que a adição de frutas tropicais na elaboração de sorvetes desempenha papel fundamental tanto nas características finais do produto como na aceitação dos consumidores. Os objetivos deste trabalho foram caracterizar físico-quimicamente as polpas de duas variedades de tamarillo e caracterizar físico-química e sensorialmente quatro formulações de sorvetes elaborados com a polpa do fruto. Para caracterização da polpa de fruta e do sorvete, foram realizadas análises de umidade, cinzas, carboidratos, proteína, gordura, acidez titulável, textura e overrun. Os sorvetes foram analisados sensorialmente para os atributos de cor, sabor, textura, impressão global e intenção de compra. Os parâmetros físico-químicos apresentaram diferenças tanto entre as variedades de tamarillo quanto entre as diferentes formulações de sorvete, exceto no teor proteico do fruto. Os valores de overrun dos sorvetes elaborados variaram de $66,41 \%$ a $92,30 \%$. O sorvete adicionado de $20 \%$ de polpa de tamarillo roxo obteve a melhor nota no atributo sabor, 7,85 . No geral, os produtos obtiveram boa aceitação. Conclui-se que a concentração e a variedade da polpa utilizada influenciam nas características do sorvete e que o tamarillo pode ser considerado uma fruta em potencial na fabricação do produto.
\end{abstract}

Palavras-chave: Tomate de árvore; Polpa de fruta; Gelado comestível; Processamento; Fruta exótica; Leite; Composição. 


\begin{abstract}
Also known in Brazil as tree tomatoes, tamarillo fruit has gained prominence due to the richness of the nutrients and bioactive compounds that it presents, being a promising candidate for application by the food industry. It is emphasized that the addition of tropical fruits in the preparation of ice cream plays a key role, both in the final characteristics of the product as acceptance of the consumers. This work aimed to characterize, physical and chemically, the pulps of two varieties of tamarillo and to characterize, physical, chemical, and sensorially, four ice cream formulations made with the fruit pulp. To characterize the fruit pulp and ice cream, moisture, ash, carbohydrates, protein, fat, titratable acidity, texture, and overrun analyzes were performed. The ice creams were analyzed sensorially for the attributes of color, flavor, texture, global impression, and purchase intention. The physicochemical parameters showed differences both between the varieties of tamarillo and the different ice cream formulations, except in the fruit protein content. The overrun values of the prepared ice creams ranged from $66.41 \%$ to $92.30 \%$. The ice cream added with purple tamarillo pulp at $20 \%$ obtained the best score in the flavor attribute, 7.85. In general, the products obtained good acceptance. It is concluded that the concentration and variety of the pulp used to influence the ice cream characteristics and that the tamarillo can be considered a potential fruit in the product manufacture.
\end{abstract}

Keywords: Tree tomato; Fruit pulp; Iced food; Processing; Exotic fruit; Milk; Composition.

\title{
1 Introdução
}

O tamarillo, também conhecido como tomate de árvore (Solanum betacea Cav.) é um importante fruto dos Andes e sua origem exata permanece desconhecida. No final do século 19, a fruta foi introduzida globalmente na Oceania, no Sudeste Asiático, na Europa e na África. Atualmente, apenas três países, Austrália, Colômbia e Nova Zelândia, cultivam tamarillo comercialmente (Diep et al., 2020; Ramírez \& Kallarackal, 2019). No Brasil, é pouco cultivado, restringindo-se a produções domésticas das três variedades existentes (amarela, roxa e vermelha), principalmente nos estados Bahia, Minas Gerais e São Paulo (Pantoja et al., 2009). De acordo com Acosta-Quezada et al. (2015), a cor dos frutos é determinada pela presença de clorofila, carotenoides e antocianinas.

O tamarillo possui diversos nutrientes, como fibras dietéticas, polifenóis, vitaminas $\mathrm{C}, \mathrm{A}, \mathrm{B}$ e $\mathrm{E}$, carotenoides, potássio e ferro. Os efeitos do tamarillo na saúde incluem propriedades antioxidantes, antiproliferativas, antinociceptivas, anti-inflamatórias, antialérgicas e antiobesas (Wang \& Zhu, 2020). Nos últimos anos, pesquisas demonstraram diferentes propriedades e utilidades do tamarillo (Diep et al., 2020; Gannasin et al., 2015; Paes et al., 2015; Santander et al., 2017), o que o torna um candidato promissor no setor alimentício e na indústria de gelados comestíveis.

O sorvete é considerado alimento de boa aceitação sensorial, alta densidade calórica e nutricional. Além dos ingredientes básicos da sua composição, é possível adicionar frutas tropicais, as quais podem influenciar em diversas características finais do produto, como palatabilidade, textura, cor, entre outras (ElSamahy et al., 2009; Souza et al., 2010). Muitas frutas exóticas, devido às propriedades benéficas, têm alto potencial de consumo e demanda (Acosta-Quezada et al., 2015), e, se adicionadas ao sorvete, podem enriquecer o produto, além de aumentar as opções de sabores e aromas disponibilizados aos consumidores (Yangilar, 2015).

No entanto, mudanças na formulação ou nas condições do processo frequentemente causam alterações estruturais no sorvete, influenciando nas características reológicas, físico-químicas e sensoriais do produto (Muse \& Hartel, 2004). Conforme reportado por Soares et al. (2018), a adição de diferentes concentrações de polpa de jambolão afeta as propriedades do sorvete, como overrun, cor e aceitação sensorial, corroborando com os relatos de Stanley et al. (1996), que afirmam que a escolha da melhor formulação do sorvete é necessária para obtenção de características desejáveis, inclusive de melhoria da estrutura física do produto. 
Esta pesquisa teve como objetivos avaliar as características físico-químicas da polpa de tamarillo e analisar as características físicas, químicas e sensoriais de quatro formulações de sorvete elaborado com a incorporação deste fruto.

\section{Material e métodos}

\subsection{Obtenção da polpa de tamarillo}

As variedades roxa e amarela de tamarillo, coletadas no município de Barbacena-MG, foram utilizadas neste experimento. Os processos de obtenção da polpa e produção de sorvete foram realizados no Laboratório de Nutrição do Instituto Federal do Sudeste de Minas Gerais, Campus Barbacena.

Os frutos foram colhidos e selecionados visualmente de acordo com grau de maturação e qualidade, sendo descartados os imaturos ou com injúrias mecânicas. Após a seleção, passaram por lavagem, seguida de sanitização com água clorada a 100 ppm/15 min. Em seguida, os frutos foram submetidos à etapa de branqueamento a $100{ }^{\circ} \mathrm{C} / 10 \mathrm{~min}$, resfriados, triturados em liquidificador industrial e peneirados para separação das cascas e sementes. Posteriormente, a polpa obtida foi envasada em sacos plásticos de polietileno e congelada a $-18^{\circ} \mathrm{C}$.

\subsection{Elaboração do sorvete de tamarillo}

Foram elaboradas quatro formulações de sorvete, em concentrações de $20 \%$ e $30 \%$ de polpa, com as variedades de tamarillo roxo e amarelo (Tabela 1). Para cada formulação, os ingredientes sacarose, xarope de glicose, estabilizante/emulsificante, creme de leite, leite em pó, leite pasteurizado e pó saborizante foram homogeneizados inicialmente em liquidificador industrial para obtenção da calda. Esta calda foi pasteurizada a $65{ }^{\circ} \mathrm{C} / 30 \mathrm{~min}$, seguida de resfriamento até atingir temperatura ambiente. Posteriormente, a polpa foi adicionada e a temperatura da mistura foi ajustada para $4{ }^{\circ} \mathrm{C} / 24 \mathrm{~h}$, para promover a maturação. Todas as formulações foram submetidas às mesmas condições de congelamento em produtora descontínua horizontal, com tempo de batimento de $10 \mathrm{~min}$. Finalmente, o sorvete foi acondicionado em embalagens específicas e imediatamente armazenado a $-25^{\circ} \mathrm{C}$.

Tabela 1. Formulações dos sorvetes com polpa de tamarillo.

\begin{tabular}{ccccc}
\hline \multirow{2}{*}{ Ingrediente } & \multicolumn{3}{c}{ Formulação } \\
\cline { 2 - 4 } & $\mathbf{1 *}$ & $\mathbf{2} * *$ & $\mathbf{3}$ & $\mathbf{4} * *$ \\
\hline Sacarose & $14 \%$ & $14 \%$ & $14 \%$ & $14 \%$ \\
\hline Xarope de glicose & $4,2 \%$ & $4,2 \%$ & $4,2 \%$ & $0,2 \%$ \\
\hline Estabilizante/emulsificante & $0,7 \%$ & $0,7 \%$ & $0,7 \%$ & $4,7 \%$ \\
\hline Creme de leite & $4,7 \%$ & $4,7 \%$ & $4,7 \%$ & $1,4 \%$ \\
\hline Leite em pó & $1,4 \%$ & $1,4 \%$ & $1,4 \%$ & $43,5 \%$ \\
\hline Leite pasteurizado & $53,5 \%$ & $53,5 \%$ & $43,5 \%$ & $30 \%$ \\
\hline Polpa de tamarillo & $20 \%$ & $20 \%$ & $30 \%$ & $1,4 \%$ \\
\hline Pó saborizante (nata) & $1,4 \%$ & $1,4 \%$ & $1,4 \%$ & \\
\hline
\end{tabular}

*Polpa de tamarillo amarelo. **Polpa de tamarillo roxo. 


\subsection{Análises físico-químicas}

As análises físico-químicas da polpa e do sorvete de tamarillo foram realizadas de acordo com a Association of Official Analytical Chemists (2005): teor de umidade obtido pelo método gravimétrico; conteúdo de nitrogênio total empregando-se o método de micro-Kjedahl; conteúdo de cinzas determinado a partir da eliminação da matéria orgânica à temperatura de $550{ }^{\circ} \mathrm{C}$; lipídeos determinados pelo método de extração em Soxhlet, e os carboidratos foram quantificados pela diferença das porcentagens médias de água, proteínas, lipídeos e cinzas. A acidez titulável foi determinada por titulometria $(\mathrm{NaOH} 0,1 \mathrm{M})$, utilizando fenolftaleína como indicador, e os resultados foram expressos em porcentagem (\%) de ácido cítrico.

Os sorvetes de tamarillo formulados ainda foram submetidos à análise de coloração, firmeza e incorporação de ar. A cor foi determinada por leitura direta da reflectância dos parâmetros de cor L*, a* e $\mathrm{b}^{*}$. Os valores numéricos de $\mathrm{a}^{*} \mathrm{e} \mathrm{b}^{*}$ foram convertidos em $\mathrm{C}^{*}$ (Chroma) e Ângulo hue $\left({ }^{\circ} \mathrm{H}\right)$, de acordo com as Equações 1 e 2, respectivamente.

$$
\begin{aligned}
& H^{\circ}=\text { cotangente }\left\{\left(b^{*}\right)\right\} \\
& C=\sqrt{\left\{\left(a^{*}\right)+\left(b^{*}\right)\right\}}
\end{aligned}
$$

A análise de firmeza foi conduzida em texturômetro (TA-XT2i, Texture Tech. Corp., Scarsdale, USA), usando-se o acessório Knife Edge (HDP/BS0). As dimensões da probe foram: 0,3 cm de espessura (extremidade sem corte), $7 \mathrm{~cm}$ de comprimento e $10 \mathrm{~cm}$ de altura. Antes da realização dos testes, as amostras de sorvete foram colocadas em copos descartáveis de $50 \mathrm{~mL}$ e armazenadas em freezer a $-18^{\circ} \mathrm{C} / 24 \mathrm{~h}$. As condições de análise foram as seguintes: força de gatilho de $5 \mathrm{~g}$, distância de penetração de $50 \mathrm{~mm}$; velocidade da probe durante a penetração de $2 \mathrm{~mm} / \mathrm{s}$, velocidade da probe antes da penetração de $2 \mathrm{~mm} / \mathrm{s}$ e velocidade da probe após a penetração de $10 \mathrm{~mm} / \mathrm{s}$. Todos os dados obtidos foram analisados com o auxílio do software Exponent Lite Express (Stable Micro Systems, Godalming, UK). A firmeza foi medida como a força de compressão máxima (N) durante a penetração da probe na amostra (Aime et al., 2001).

A análise de incorporação de ar (overrun) foi realizada conforme metodologia descrita por Segall \& Goff (2002), com modificações. As amostras foram acondicionadas em béquer graduado de $250 \mathrm{~mL}$ e deixadas à temperatura ambiente até derretimento completo. Após este período, foi anotado o volume líquido resultante (calda) e, através da diferença entre os volumes final (calda) e inicial (sorvete), obteve-se a porcentagem de ar incorporado. O cálculo foi realizado conforme a Equação 3:

$$
\operatorname{Overrun}(\%)=\frac{(\text { Volumedo sorvete }- \text { Volume da calda }) \times 100}{\text { Volumeda calda }}
$$

\subsection{Caracterização sensorial do sorvete de tamarillo}

Os testes sensoriais foram realizados com aprovação prévia do Comitê de Ética em Pesquisa com Seres Humanos (CAEE: 83459917.3.0000.5156). Para os testes sensoriais, foram recrutados voluntariamente 65 consumidores pertencentes à comunidade acadêmica do Instituto Federal do Sudeste de Minas Gerais, Campus Barbacena. Os sorvetes foram submetidos à análise sensorial em que as formulações foram avaliadas por teste de aceitação quanto aos atributos de cor, sabor, textura e impressão global, utilizando escala hedônica estruturada de nove pontos, ancorada nos extremos pelos termos "desgostei muitíssimo" (1) e "gostei muitíssimo" (9) (Dantas et al., 2010). Foi empregado também teste de intenção de compra utilizando escala de atitude estruturada em cinco pontos, variando de "certamente não compraria" (1) a "certamente compraria" (5).

As amostras foram codificadas com números de três dígitos, apresentadas sob luz ambiente e oferecidas a cada um dos consumidores. As quatro amostras de sorvetes foram servidas simultaneamente à temperatura de $-10^{\circ} \mathrm{C}$, em porções de $15 \mathrm{~g}$, colocadas em copos descartáveis brancos, seguindo balanceamento de 
ordenação para apresentação das amostras (Wakeling \& MacFie, 1995). Água mineral à temperatura ambiente foi servida entre as amostras para que os consumidores pudessem efetuar a limpeza do palato.

\subsection{Análise estatística}

O experimento foi realizado em delineamento inteiramente casualizado (DIC). As análises físico-químicas das polpas e dos sorvetes foram realizadas com três repetições. Os dados obtidos no experimento foram submetidos à análise de variância (ANOVA), com utilização do teste de Scott-Knott para comparação das médias, considerando-se nível de significância de 5\%. As análises estatísticas foram realizadas com auxílio do programa estatístico R (R Core Team, 2014).

\section{Resultados e discussão}

\subsection{Caracterização físico-química da polpa de tamarillo}

As diferenças na composição do tamarillo podem ocorrer tanto na mesma cultivar quanto entre diferentes grupos (Acosta-Quezada et al., 2015). Os dois tipos de polpa apresentaram composição centesimal diferente, exceto para o teor de proteínas (Tabela 2). Ressalta-se que, em relação a estas variáveis, a polpa de tamarillo amarelo apresentou estatisticamente maior concentração de umidade $(93,06 \%)$ e cinzas $(0,96 \%)$. Em contrapartida, a polpa roxa mostrou teores superiores de lipídeos e carboidratos, 0,65\% e 5,33\%, respectivamente. Dentre os componentes, carboidratos apresentaram a maior diferença entre as cultivares, cerca de $24 \%$. Ao avaliar a acidez, observou-se que o tamarillo roxo apresentou teor $70 \%$ superior ao da variedade amarela.

Tabela 2. Valores (média \pm desvio padrão) dos teores de umidade, gorduras, cinzas, proteínas, carboidratos e acidez titulável (AT) das polpas de tamarillo, variedades amarela e roxa.

\begin{tabular}{ccccccc}
\hline \multirow{2}{*}{ Variedade } & Umidade & Lipídeos & Cinzas & Proteínas & Carboidratos & \multirow{2}{*}{ AT $^{*}$} \\
\cline { 2 - 6 } & \multicolumn{7}{c}{$\%$} & \multicolumn{1}{c}{$\%$} & \\
\hline Amarela & $93,06^{\mathrm{a}} \pm 2,21$ & $0,61^{\mathrm{b}} \pm 0,001$ & $0,96^{\mathrm{a}} \pm 0,002$ & $1,06^{\mathrm{a}} \pm 0,05$ & 4,31 & $0,91^{\mathrm{b}} \pm 0,01$ \\
\hline Roxa & $92,01^{\mathrm{b}} \pm 2,09$ & $0,65^{\mathrm{a}} \pm 0,001$ & $0,95^{\mathrm{b}} \pm 0,002$ & $1,06^{\mathrm{a}} \pm 0,05$ & 5,33 & $1,55^{\mathrm{a}} \pm 0,02$ \\
\hline
\end{tabular}

Médias seguidas com letras iguais na mesma coluna não diferem entre si, pelo teste Scott-Knott $(p>0,05)$. Acidez titulável expressa em porcentagem de ácido cítrico.

Alguns autores avaliaram a composição centesimal de tamarillo de diferentes variedades. Ao se comparar com os resultados do presente trabalho, Pantoja et al. (2009) reportaram concentrações menores de umidade, porém maiores de lipídeos, proteínas e carboidratos. Os autores ainda observaram grande discrepância no teor de cinzas entre as cultivares estudadas (amarela e vermelha), com valores sete vezes superiores $(0,15 \mathrm{e}$ 1,05\%, respectivamente). Em variedades provenientes da Nova Zelândia, Lister et al. (2005) relataram menores concentrações de umidade e lipídeos, contudo observaram resultados similares quanto ao teor de carboidratos $(4,3 \%$ e $4,6 \%)$.

O ambiente pré-colheita, que inclui clima e temperatura, juntamente com fatores de cultivo, como tipo de solo, disponibilidade de água e nutrientes, podem influenciar no conteúdo dos componentes, como proteínas e cinzas (Bastos et al., 2016). A umidade é influenciada pelas diferentes características morfológicas dos pericarpos e estádio de amadurecimento dos frutos, processo que pode levar à desidratação e, como resultado, à maior concentração de outros constituintes (Lago-Vanzela et al., 2011). Os lipídeos são moléculas altamente energéticas e aparecem normalmente em pequenas quantidades em frutas (Lago-Vanzela et al., 2011), como pode ser constatado neste trabalho. Tais resultados indicam ocorrer uma grande variação do teor 
lipídico entre as diferentes variedades de tamarillo e distintas regiões produtoras. De acordo com Mesa et al. (2016), diferentes práticas culturais alteram a dinâmica de alocação de carboidratos e, consequentemente, ocorre a variação deste componente.

A Base de Dados de Composição de Alimentos da Nova Zelândia detalha a composição aproximada do tamarillo. De acordo com os valores tabelados, o fruto apresenta teores de umidade, lipídeos, proteínas e carboidratos de 86,1, 0,4,2,0 e 3,8\%, respectivamente (Sivakumaran et al., 2015). Comparando-se os dados, verifica-se que os tamarillos do nosso estudo apresentam, com exceção do teor proteico, teores mais elevados de todos os componentes mencionados.

Os valores de acidez provenientes da variedade amarela foram inferiores aos reportados por Pantoja et al. (2009) e por Meza \& Manzano Méndez (2009), que observaram, respectivamente, concentração de 1,65 e $1,20 \%$ de ácido cítrico para a mesma cultivar. Reforça-se que o grau de maturação influencia na acidez total, uma vez que o fruto, em estádios iniciais, apresenta maior teor de ácidos (Aroucha et al., 2012).

\subsection{Caracterização físico-química do sorvete de tamarillo}

As formulações testadas obtiveram diferenças em todos os componentes avaliados e os maiores valores de umidade e cinzas, e o menor teor de carboidratos foram observados na formulação 4 (Tabela 3). Além disso, a formulação 3 apresentou estatisticamente os menores valores de lipídeos e umidade, porém maior porcentual de carboidratos. Esses resultados provavelmente estão relacionados à proporção da polpa utilizada e à redução do leite pasteurizado na elaboração do sorvete. Finalmente, a formulação 1 obteve os menores valores de cinzas, enquanto a formulação 2, o menor teor de proteínas.

Tabela 3. Teores, expressos em porcentagem, de umidade, cinzas, gorduras, lipídeos, proteínas, carboidratos e acidez titulável (AT) das quatro formulações de sorvete com polpa de tamarillo.

\begin{tabular}{ccccccc}
\hline Formulação & Umidade & Cinzas & Lipídeos & Proteínas & Carboidratos & AT \\
\hline 1 & $69,83^{\mathrm{b}} \pm 0,09$ & $0,65^{\mathrm{c}} \pm 0,003$ & $1,52^{\mathrm{a}} \pm 0,02$ & $3,25^{\mathrm{a}} \pm 0,02$ & $24,75^{\mathrm{c}} \pm 0,04$ & $0,28^{\mathrm{d}} \pm 0,004$ \\
\hline 2 & $68,42^{\mathrm{c}} \pm 0,06$ & $0,66^{\mathrm{b}} \pm 0,003$ & $1,54^{\mathrm{a}} \pm 0,02$ & $3,05^{\mathrm{c}} \pm 0,01$ & $26,33^{\mathrm{b}} \pm 0,04$ & $0,35^{\mathrm{c}} \pm 0,005$ \\
\hline 3 & $67,48^{\mathrm{d}} \pm 0,06$ & $0,66^{\mathrm{b}} \pm 0,003$ & $1,25^{\mathrm{b}} \pm 0,01$ & $3,17^{\mathrm{b}} \pm 0,02$ & $27,44^{\mathrm{a}} \pm 0,04$ & $0,37^{\mathrm{b}} \pm 0,005$ \\
\hline 4 & $70,89^{\mathrm{a}} \pm 0,07$ & $0,82^{\mathrm{a}} \pm 0,004$ & $1,52^{\mathrm{a}} \pm 0,02$ & $3,22^{\mathrm{a}} \pm 0,01$ & $23,55^{\mathrm{d}} \pm 0,03$ & $0,39^{\mathrm{a}} \pm 0,005$ \\
\hline
\end{tabular}

Médias seguidas de letras iguais na coluna não diferem entre si pelo teste de Scott-Knott $(p>0,05)$. Formulação 1: 20\% polpa do tamarillo amarelo; Formulação 2: 20\% polpa de tamarillo roxo; Formulação 3: 30\% polpa de tamarillo amarelo; Formulação 4: 30\% polpa de tamarillo roxo.

De acordo com Morzelle et al. (2012), o conteúdo de lipídeos auxilia no desenvolvimento da textura, do corpo e dos atributos sensoriais do sorvete, e pode ser atribuído tanto à polpa do fruto quanto ao leite utilizados na fabricação do produto (Souza et al., 2010). O tamarillo possui quantidade pouco destacável de lipídeos $(<1,0 \%)$ e, consequentemente, a adição da fruta tem pouca representatividade na fração lipídica do produto final.

Há relatos na literatura de que a polpa do tamarillo pode apresentar proteína bruta em níveis de até 9,6\% (Wang \& Zhu, 2020), o que contribuiria nas propriedades de textura, estrutura e viscosidade do sorvete (Cheng et al., 2015; Souza et al., 2010). Contudo, os dados reportados na literatura sobre a composição proteica da polpa de tamarillo divergem dos observados no presente estudo, no qual os valores foram de aproximadamente $1,0 \%$ (Tabela 2 ).

O teor de carboidratos presente nos sorvetes é proveniente do somatório de diversos ingredientes, como a porcentagem de açúcar da polpa, da lactose do leite e da sacarose comercial adicionada. Além disso, convém ressaltar que os açúcares contribuem para redução do ponto de congelamento e para aumento da viscosidade 
e do tempo de batimento da mistura, bem como da cremosidade e do sabor (Morzelle et al., 2012; Souza et al., 2010).

Em relação à acidez, os maiores teores de ácido cítrico foram observados nas formulações com maior concentração de polpa (Tabela 4), uma vez que o tamarillo é notadamente rico em ácidos. A formulação 4 ( $30 \%$ de polpa roxa) apresentou estatisticamente a maior acidez, um resultado coerente, pois a polpa de tamarillo roxa também apresentou os maiores teores (Tabela 3). Ao se comparar com os resultados do presente estudo, níveis de acidez inferiores (Fidelis et al., 2015) e superiores (Lamounier et al., 2014) foram reportados em sorvetes elaborados com adição de diferentes frutos, o que reforça a influência da matériaprima nas características finais do produto.

A textura do sorvete é resultado da sua estrutura e depende de vários fatores presentes, tais como o estado de agregação dos glóbulos de gordura, a quantidade de ar incorporado, o tamanho das bolhas de ar e dos cristais de gelo, e as macromoléculas e os solutos dissolvidos e suspensos (Akalın et al., 2008; Goff, 1997; Souza et al., 2010; Varela et al., 2014). Em nosso estudo, o porcentual de polpa adicionada influenciou estatisticamente $(p>0,05)$ na textura (firmeza) do sorvete (Tabela 4), ou seja, formulações com $20 \%$ de polpa obtiveram menor textura que as formulações com $30 \%$ de polpa. De acordo com Abdel-Haleem \& Awad (2015), o aumento significativo na textura dos sorvetes com maior acréscimo de polpa pode ser atribuído ao maior teor de fibra alimentar, a qual pode atuar como estabilizante e reduzir o teor de água livre. Resultados similares ao presente estudo foram reportados em sorvetes elaborados à base de pequi e curriola, porém os sorvetes produzidos com araticum ou mangaba apresentaram valores inferiores de textura (Morzelle et al., 2012).

A incorporação de ar, definida como o aumento do volume do sorvete obtido a partir de um volume inicial de calda, é expressa em porcentagem de overrun (Souza et al., 2010). Adicionalmente, os equipamentos utilizados no processo influenciam nas propriedades do produto, como o uso dos congeladores descontínuos, os quais geram menor incorporação de ar quando comparados aos congeladores contínuos (Souza et al., 2010; Vacondio et al., 2013). Os valores de overrun dos sorvetes de tamarillo variaram entre $66,41 \% \mathrm{e}$ $92,30 \%$, ocorrendo decréscimo com o aumento da concentração da polpa (Tabela 4). Sugere-se que este aumento da concentração da polpa de tamarillo diminuiu a incorporação de ar provavelmente devido ao $\mathrm{pH}$ reduzido/maior acidez (Tabela 3) dos sorvetes, o que pode ter afetado a estabilidade da emulsão (Soares et al., 2018).

Resultados semelhantes de overrun foram observados por Lamounier et al. (2014), em análise de sorvete de açaí, banana e guaraná. O presente trabalho também corrobora com os dados obtidos por Campidelli et al. (2015), que verificaram $71,91 \%$ de incorporação de ar em sorvete elaborado com mirtilo. Em contraste, porcentagens menores, entre $16,0 \%$ e $19,0 \%$, foram relatadas por Campidelli et al. (2015) em sorvetes de iogurte adicionados de polpa de açaí. Sofjan \& Hartel (2004) verificaram correlação negativa entre firmeza e overrun nas diferentes amostras de sorvetes analisadas, com resultados semelhantes aos deste trabalho, visto que as formulações com maior textura apresentaram menor overrun.

Em relação aos parâmetros de cor, a formulação 4 (30\% de polpa de tamarillo roxo), por apresentar estatisticamente menor ângulo hue $\left({ }^{\circ} \mathrm{H}\right)$, pode ser considerada levemente mais avermelhada que as demais, seguida pela formulação 2 ( $20 \%$ de polpa de tamarillo roxo), que apresenta uma coloração mais próxima ao laranja. Por outro lado, as formulações 1 e 3, ambas contendo polpa de tamarillo amarelo, apresentaram valores de ${ }^{\circ} \mathrm{H}$ estatisticamente iguais, indicando coloração mais próxima ao amarelo (Tabela 4). Ressalta-se que as diferenças da coloração vermelha das formulações contendo o tamarillo roxo devem-se pela intensa característica da polpa e pela proporção utilizada, assim como houve maior intensidade da coloração amarela nas formulações que continham polpa de tamarillo amarelo. No presente estudo, os dados mostram baixa variação de ${ }^{\circ} \mathrm{H}$ nas amostras, em contraste com os resultados reportados por Topdaş et al. (2017), que constataram diminuição considerável de ${ }^{\circ} \mathrm{H}$ à medida que aumentavam a concentração do fruto adicionado ao sorvete. 
Tabela 4. Valores (médias \pm desvio padrão) das análises de textura, porcentagem de overrun e dos parâmetros de cor Chroma e ângulo Hue $\left({ }^{\circ} \mathrm{H}\right)$ das quatro formulações de sorvete com polpa de tamarillo.

\begin{tabular}{ccccc}
\hline \multirow{2}{*}{ Formulação } & \multicolumn{4}{c}{ Parâmetro } \\
\cline { 2 - 5 } & Textura (N) & Overrun & Croma & 'Hue \\
\hline 1 & $19,61^{\mathrm{b}} \pm 0,31$ & $85,18 \%$ & $29.79^{\mathrm{b}} \pm 0,13$ & $78.14^{\mathrm{a}} \pm 0,33$ \\
\hline 2 & $15,44^{\mathrm{b}} \pm 0,25$ & $92,30 \%$ & $28.07^{\mathrm{b}} \pm 0,12$ & $71.53^{\mathrm{b}} \pm 0,30$ \\
\hline 3 & $27,38^{\mathrm{a}} \pm 0,44$ & $72,41 \%$ & $32.06^{\mathrm{a}} \pm 0,14$ & $78.06^{\mathrm{a}} \pm 0,33$ \\
\hline 4 & $31,36^{\mathrm{a}} \pm 0,09$ & $66,41 \%$ & $27.80^{\mathrm{b}} \pm 0,12$ & $66.63^{\mathrm{c}} \pm 0,28$ \\
\hline
\end{tabular}

Médias seguidas de letras iguais na coluna não diferem entre si pelo teste de Scott-Knott $(p>0,05)$. Formulação 1: 20\% polpa do tamarillo amarelo; Formulação 2: 20\% polpa de tamarillo roxo; Formulação 3: 30\% polpa de tamarillo amarelo; Formulação 4: 30\% polpa de tamarillo roxo.

O índice croma define a saturação da coloração dos frutos, sendo que valores mais elevados de $\mathrm{C}^{*}$ indicam maior intensidade da cor (Pathare et al., 2013) e tendência de homogeneidade da coloração no produto (Fernandes et al., 2015). Em relação ao parâmetro Chroma, os valores variaram de 27,80 a 32,06, com maior valor apresentado pela formulação 3, não diferindo estatisticamente entre si as formulações 1,2 e 4.

Finalmente, este estudo corrobora com diversos achados que comprovaram que as propriedades dos sorvetes são influenciadas pelas espécies ou cultivares frutíferas utilizadas, assim como pela proporção da polpa adicionada (Hamad et al., 2017; Morzelle et al., 2012; Soares et al., 2018).

\subsection{Caracterização sensorial do sorvete de tamarillo}

De acordo com Wang \& Zhu (2020), o tamarillo é um fruto com potencial a ser explorado, sendo promissor na elaboração de produtos saudáveis. De fato, pelos testes sensoriais, percebe-se esta potencialidade, visto que todos os sorvetes elaborados apresentaram pontuações relativamente elevadas para todas as características avaliadas (Tabela 5).

Tabela 5. Propriedades sensoriais dos sorvetes elaborados com quatro formulações de sorvete com polpa de tamarillo.

\begin{tabular}{cccccc}
\hline Formulação & Cor & Sabor & Textura & Impressão global & Intenção de compra \\
\hline 1 & $6,83^{\mathrm{b}}$ & $6,94^{\mathrm{b}}$ & $7,58^{\mathrm{a}}$ & $7,24^{\mathrm{b}}$ & $3,46^{\mathrm{b}}$ \\
\hline 2 & $7,65^{\mathrm{a}}$ & $7,85^{\mathrm{a}}$ & $7,98^{\mathrm{a}}$ & $7,86^{\mathrm{a}}$ & $4,13^{\mathrm{a}}$ \\
\hline 3 & $7,46^{\mathrm{a}}$ & $7,17^{\mathrm{b}}$ & $7,78^{\mathrm{a}}$ & $7,40^{\mathrm{b}}$ & $3,71^{\mathrm{b}}$ \\
\hline 4 & $7,82^{\mathrm{a}}$ & $7,15^{\mathrm{b}}$ & $7,66^{\mathrm{a}}$ & $7,64^{\mathrm{a}}$ & $4,00^{\mathrm{a}}$ \\
\hline
\end{tabular}

Médias seguidas de letras iguais na mesma coluna não diferem entre si pelo teste de Scott-Knott $(p>0,05)$. Formulação 1: $20 \%$ polpa do tamarillo amarelo; Formulação 2: 20\% polpa de tamarillo roxo; Formulação 3: 30\% polpa de tamarillo amarelo; Formulação 4: $30 \%$ polpa de tamarillo roxo.

Os dados mostram que a maior parte das médias de aceitação foi acima de 7, situando-se, na escala hedônica, entre os termos "gostei moderadamente" e "gostei muito". As médias de intenção de compra situam-se entre "talvez comprasse/talvez não comprasse" e "possivelmente compraria". Este resultado mostra que, mesmo produzido com polpa de fruta não convencional, o sorvete alcançou boa aceitação.

De forma geral, as maiores médias de aceitação, para todos os parâmetros avaliados, foram observadas para os sorvetes formulados com a polpa de tamarillo roxo, com destaque para a formulação com $20 \%$, que obteve média no atributo sabor superior às demais formulações e apresentou impressão global e intenção de compra estatisticamente iguais ao sorvete com $30 \%$ de polpa roxa. 
Elevadas pontuações também foram relatadas por Goraya \& Bajwa (2015), em sorvetes com diferentes concentrações de polpa de groselha indiana, nos quais obtiveram valores dos atributos sensoriais variando de 6,25 a 8,50. Resultados similares foram reportados por Yuksel (2015), que observou valores de algumas propriedades sensoriais entre 6,70 e 8,02 , para sorvetes com porcentagens distintas de abrunho. Lamounier et al. (2014) obtiveram médias dos atributos sensoriais relativamente elevadas em sorvete com açaí, guaraná e banana, todas acima de 8 , além de o produto ter apresentado nota 4,5 no quesito sobre a intenção de compra.

\section{Conclusões}

A concentração da polpa e a cultivar utilizada influenciaram as características físico-químicas dos sorvetes.

Os resultados apresentados mostraram que é possível produzir sorvete de tamarillo com características desejáveis. Notadamente, a cultivar roxa se desponta promissora neste cenário, devido aos bons resultados físico-químicos e sensoriais dos sorvetes elaborados com essa variedade.

Portanto, esta fruta, pouco conhecida no Brasil, poderá ser considerada como potencial ingrediente na indústria de sorvetes.

\section{Referências}

Abdel-Haleem, A. M., \& Awad, R. A. (2015). Some quality attributes of low fat ice cream substituted with hulless barley flour and barley ß-glucan. Journal of Food Science and Technology, 52(10), 6425-6434. PMid:26396387. http://dx.doi.org/10.1007/s13197-015-1755-x

Acosta-Quezada, P. G., Raigón, M. D., Riofrío-Cuenca, T., García-Martínez, M. D., Plazas, M., Burneo, J. I., Figueroa, J. G., Vilanova, S., \& Prohens, J. (2015). Diversity for chemical composition in a collection of different varietal types of tree tomato (Solanum betaceum Cav.), an Andean exotic fruit. Food Chemistry, 169, 327-335. PMid:25236234. http://dx.doi.org/10.1016/j.foodchem.2014.07.152

Aime, D. B., Arntfield, S. D., Malcolmson, L. J., \& Ryland, D. (2001). Textural analysis of fat reduced vanilla ice cream products. Food Research International, 34(2-3), 237-246. http://dx.doi.org/10.1016/S0963-9969(00)00160-5

Akalın, A. S., Karagözlü, C., \& Ünal, G. (2008). Rheological properties of reduced-fat and low-fat ice cream containing whey protein isolate and inulin. European Food Research and Technology, 227(3), 889-895. http://dx.doi.org/10.1007/s00217-0070800-z

Aroucha, E. M. M., Souza, C. S. M., Souza, A. E. D., Ferreira, R. M. A., \& Aroucha Filho, J. C. (2012). Qualidade pós-colheita da cajarana em diferentes estádios de maturação durante armazenamento refrigerado. Revista Brasileira de Fruticultura, 34(2), 391-399. http://dx.doi.org/10.1590/S0100-29452012000200011

Association of Official Analytical Chemists - AOAC. (2005). Association of Official and Analytical Chemists (17th ed.). Arlington: AOAC.

Bastos, J. S., Martinez, E. A., \& Souza, S. M. A. (2016). Características físico-químicas da polpa de umbu (Spondias tuberosa Arruda Câmara) comercial: Efeito da concentração. Journal of Bioenergy and Food Science, 3(1), 11-16. http://dx.doi.org/10.18067/jbfs.v3i1.48

Campidelli, M. L. L., Paulinelli, H. R., Magalhães, M. L., Penoni, N., \& Carlos, F. G. (2015). Efeitos do enriquecimento da semente de chia (Salvia hispanica) nas propriedades de sorvete de mirtilo (Vaccinium myrtillus). Revista Brasileira de Tecnologia Agroindustrial, 9(2), 1962-1974. http://dx.doi.org/10.3895/rbta.v9n2.1820

Cheng, J., Ma, Y., Li, X., Yan, T., \& Cui, J. (2015). Effects of milk protein-polysaccharide interactions on the stability ofice cream mix model systems. Food Hydrocolloids, 45, 327-336. http://dx.doi.org/10.1016/j.foodhyd.2014.11.027

Dantas, M. I. D. S., Silva, M. G. D., Pinto, C. R. R., Pereira, C. A. D. S., Minim, V. P. R., \& Bittencourt, M. C. B. (2010). Farinhas de soja sem lipoxigenase agregam valor sensorial em bolos. Revista Ceres, 57(2), 141-144. http://dx.doi.org/10.1590/S0034737X2010000200001

Diep, T. T., Rush, E. C., \& Yoo, M. J. Y. (2020). Tamarillo (Solanum betaceum Cav.): A review of physicochemical and bioactive properties and potential applications. Food Reviews International. In press. http://dx.doi.org/10.1080/87559129.2020.1804931

El-Samahy, S. K., Youssef, K. M., \& Moussa-Ayoub, T. E. (2009). Producing ice cream with concentrated cactus pear pulp: A preliminary study. Journal of the Professional Association for Cactus Development, 11, 1-12.

Fernandes, A. P. S., Costa, J. B., Soares, D. S. B., Moura, C. J., \& Souza, A. R. M. (2015). Aplicação de filmes biodegradáveis produzidos a partir de concentrado proteico de soro de leite irradiado. Pesquisa Agropecuária Tropical, 45(2), $192-199$. http://dx.doi.org/10.1590/1983-40632015v4530844

Fidelis, V. R. L., Pereira, E. M., Silva, W. P., Gomes, J. P., \& Silva, L. A. (2015). Produção de sorvetes e iogurtes a partir dos frutos figo da índia e mandacaru. Revista Verde de Agroecologia e Desenvolvimento Sustentável, 10(4), 17-21. http://dx.doi.org/10.18378/rvads.v10i4.3698 
Gannasin, S. P., Mustafa, S., Adzahan, N. M., \& Muhammad, K. (2015). In vitro prebiotic activities of tamarillo (Solanum betaceum Cav.) hydrocolloids. Journal of Functional Foods, 19, 10-19. http://dx.doi.org/10.1016/j.jff.2015.09.004

Goff, H. D. (1997). Colloidal aspects of ice cream: A review. International Dairy Journal, 7(6-7), 363-373. http://dx.doi.org/10.1016/S0958-6946(97)00040-X

Goraya, R. K., \& Bajwa, U. (2015). Enhancing the functional properties and nutritional quality of ice cream with processed amla (Indian gooseberry). Journal of Food Science and Technology, 52(12), 7861-7871. PMid:26604358. http://dx.doi.org/10.1007/s13197-015-1877-1

Hamad, M. N. E. F., Nekshara, H. H., Shasta, A. S., \& Tarabia, D. S. (2017). Effect of Addition Dates "Hayani" on Yield, Chemical Composition and Sensory Evaluation of Ice Cream. American Journal of Food Science and Nutrition Research, 4(5), 170-176.

Lago-Vanzela, E. S., Ramin, P., Umsza-Guez, M. A., Santos, G. V., Gomes, E., \& Silva, R. (2011). Chemical and sensory characteristics of pulp and peel "cajá-manga" (Spondias cytherea Sonn.) jelly. Food Science and Technology, 31(2), 398-405. http://dx.doi.org/10.1590/S0101-20612011000200018

Lamounier, M. L., Silva, F. A., De Almeida, C. C., \& Leal, R. (2014). Desenvolvimento e caracterização de sorvete de açaí, guaraná e banana enriquecido com fitoesterol. Revista Brasileira de Tecnologia Agroindustrial, 8(2S), 1570-1578. http://dx.doi.org/10.3895/S1981-36862014000200010S1

Lister, C. E., Morrison, S. C., Kerkhofs, N. S., \& Wright, K. M. (2005). The nutritional composition and health benefits of New Zealand tamarillos. Crop \& Food Research Confidential Report, (1281), 29.

Mesa, K., Serra, S., Masia, A., Gagliardi, F., Bucci, D., \& Musacchi, S. (2016). Seasonal trends of starch and soluble carbohydrates in fruits and leaves of 'Abbé Fétel' pear trees and their relationship to fruit quality parameters. Scientia Horticulturae, 211, 60-69. http://dx.doi.org/10.1016/j.scienta.2016.08.008

Meza, N., \& Manzano Méndez, J. (2009). Características del fruto de tomate de árbol (Cyphomandra betaceae [Cav.] Sendtn) basadas en la coloración del arilo, en la Zona Andina Venezolana. UDO Agricola, 9(2), 289-294.

Morzelle, M. C., Lamounier, M. L., Souza, E. C., Salgado, J. M., \& Vilas-Boas, E. V. de B. (2012). Nutritional and sensory characteristics of ice cream from savana fruits. Revista do Instituto de Latícinios Cândido Tostes, 67(387), 70-78. http://dx.doi.org/10.5935/2238-6416.20120052

Muse, M. R., \& Hartel, R. W. (2004). Ice cream structural elements that affect melting rate and hardness. Journal of Dairy Science, 87(1), 1-10. PMid:14765804. http://dx.doi.org/10.3168/jds.S0022-0302(04)73135-5

Paes, A. S., Quast, L. B., Quast, E., \& Raupp, D. da S. (2015). Development of Tamarillo Light Jelly with High Pulp Content. Revista Ciencias Exatas e Naturais, 17(2), 293-306. http://dx.doi.org/10.5935/RECEN.2015.02.06

Pantoja, L., Pinto, N. A. V. D., Lopes, C., Grandra, R., \& Santos, A. S. (2009). Caracterização física e físico-química de frutos de duas variedades de tamarillo oriundas do Norte de Minas Gerais. Revista Brasileira de Fruticultura, 31(3), 916-919. http://dx.doi.org/10.1590/S0100-29452009000300041

Pathare, P. B., Opara, U. L., \& Al-Said, F. A. J. (2013). Colour measurement and analysis in fresh and processed foods: A review. Food and Bioprocess Technology, 6(1), 36-60. http://dx.doi.org/10.1007/s11947-012-0867-9

R Core Team. (2014). R: A language and environment for statistical computing. Vienna: R Foundation for Statistical Computing Ramírez, F., \& Kallarackal, J. (2019). Tree tomato (Solanum betaceum Cav.) reproductive physiology: A review. Scientia Horticulturae, 248, 206-215. http://dx.doi.org/10.1016/j.scienta.2019.01.019

Santander, M., Osorio, O., \& Mejíga, D. (2017). Evaluación de propiedades antioxidantes y fisicoquímicas de una bebida mixta durante almacenamiento refrigerado. Revista de Ciencias Agricolas, 34(1), 84-97. http://dx.doi.org/10.22267/rcia.173401.65

Segall, K. I., \& Goff, H. D. (2002). A modified ice cream processing routine that promotes fat destabilization in the absence of added emulsifier. International Dairy Journal, 12(12), 1013-1018. http://dx.doi.org/10.1016/S0958-6946(02)00117-6

Sivakumaran, S., Huffman, L., Sivakumaran, S., \& Athar, N. (2015). The concise New Zealand food composition tables. New Zealand Institute for Plant \& Food Research Limited.

Soares, J. C., Garcia, M. C., Garcia, L. G. C., Caliari, M., \& Soares Júnior, M. S. (2018). Jambolan sherbets overrun, color, and acceptance in relation to the sugar, milk, and pulp contents in formulation. Food Science and Technology (Campinas), 38(Supl.1), 313-318. http://dx.doi.org/10.1590/fst.21817

Sofjan, R. P., \& Hartel, R. W. (2004). Effects of overrun on structural and physical characteristics of ice cream. International Dairy Journal, 14(3), 255-262. http://dx.doi.org/10.1016/j.idairyj.2003.08.005

Souza, J. C. B., Costa, M. de R., Rensis, M. V. B., \& Sivieri, C. (2010). Sorvete: Composição, processamento e viabilidade da adição de probiótico. Alimentos e Nutrição, 21(1), 155-165.

Stanley, D. W., Goff, H. D., \& Smith, A. K. (1996). Texture-structure relationships in foamed dairy emulsions. Food Research International, 29(1), 1-13. http://dx.doi.org/10.1016/0963-9969(95)00063-1

Topdaş, E. F., Çakmakçi, S., \& Çakiroğlu, K. (2017). The antioxidant activity, vitamin c contents, physical, chemical and sensory properties of ice cream supplemented with cornelian cherry (Cornus mas L.) paste. Kafkas Üniversitesi Veteriner Fakültesi Dergisi, 23(5), 691-697. http://dx.doi.org/10.9775/kvfd.2016.17298

Vacondio, R., Lopes, E. S., Rosa, N. C., Carvalho, A. R., Pieretti, G. G., \& Madrona, G. S. (2013). Caracterização e avaliação sensorial de sorvete com extrato aquoso de yacon. E-Xacta, 6(2), 155. http://dx.doi.org/10.18674/exacta.v6i2.1046

Varela, P., Pintor, A., \& Fiszman, S. (2014). How hydrocolloids affect the temporal oral perception of ice cream. Food Hydrocolloids, 36, 220-228. http://dx.doi.org/10.1016/j.foodhyd.2013.10.005 
Propriedades físico-químicas da polpa de tamarillo (Solanum betaceum) e sua aplicabilidade na elaboração de sorvetes

Fernandino, C. M. et al.

Wakeling, I. N., \& MacFie, H. J. H. (1995). Designing consumer trials balanced for first and higher orders of carry-over effect when only a subset of k samples from t may be tested. Food Quality and Preference, 6(4), 299-308. http://dx.doi.org/10.1016/0950-3293(95)00032-1

Wang, S., \& Zhu, F. (2020). Tamarillo (Solanum betaceum): Chemical composition, biological properties, and product innovation. Trends in Food Science \& Technology, 95, 45-58. http://dx.doi.org/10.1016/j.tifs.2019.11.004

Yangilar, F. (2015). Effects of green banana flour on the physical, chemical and sensory properties of ice cream. Food Technology and Biotechnology, 53(3), 315-323. PMid:27904363.

Yuksel, A. K. (2015). The effects of blackthorn (Prunus spinosa L.) addition on certain quality characteristics of ice cream. Journal of Food Quality, 38(6), 413-421. http://dx.doi.org/10.1111/jfq.12170 